\title{
Increasing Physical Exercise Levels
}

\section{Age-Specific Benefits of Planning}

\author{
Urte Scholz, PhD \\ Universität Zürich, Switzerland \\ Falko F. Sniehotta, PhD \\ University of Aberdeen \& Aberdeen Centre for \\ Energy Regulation and Obesity Research, Scotland \\ Silke Burkert, Dipl-Psych \\ Charité Berlin, Germany \\ Ralf Schwarzer, $\mathrm{PhD}$ \\ Freie Universität Berlin, Germany
}

\begin{abstract}
Purpose: This study examines the differential age effects on physical exercise of two planning interventions, action planning (when, where, how) and coping planning (anticipating barriers, mental simulation of success scenarios), and examines the mediating mechanisms of the interventions. Methods: The study assigned the participants, 205 cardiac rehabilitation patients, to one of the intervention groups (action-planning only or combined-planning group) or to a control group. Baseline measurement and follow-up took place 2 months apart. Results: The interventions enhanced physical exercise independently of age. Pretreatment coping planning was higher in older (65-82 years) than in younger (38-54 years) or middle-aged (55-64 years) participants. At Time 2, older participants were the only ones without further increase in coping planning. Advancement in coping planning partially mediated the effect of the intervention. Conclusion: Coping planning facilitates improvement of physical exercise. Implications of age differences in planning are discussed.
\end{abstract}

Keywords: planning; physical exercise; self-regulation; cardiac rehabilitation; aging

\footnotetext{
Tn 2002, almost $30 \%$ of all deaths in the United States were caused by diseases of the heart (Anderson \& Smith, 2005). Similar figures were found in other Western countries (Weidner, 2001). Coronary heart disease (CHD), the most common cardiovascular disease, is mainly caused by
} 
unhealthy lifestyle behaviors, such as smoking, physical inactivity, and unhealthy diet (Merrill \& Verbrugge, 1999). Thus, CHD patients need to change their lifestyle habits to improve their risk profile. This study focuses on physical activity as an important factor in the prevention and rehabilitation of CHD (Ades, 2001). There is compelling evidence that regular physical exercise is related to lower mortality, lower relapse rates, and reduced symptoms after the manifestation of CHD (cf. Thompson et al., 2003). For example, Jolliffe and colleagues (2003) reported in a meta-analysis that exercise interventions led to a $31 \%$ reduction of total cardiac mortality in CHD patients who underwent a long-term supervised exercise training program. Thus, coronary rehabilitation patients are advised to engage in regular vigorous exercise to reduce their risk status and support the rehabilitation process (Smith, et al., 2001). Recommendations for engaging in regular physical activity are independent of a patient's age (Thompson et al., 2003). The prevalence of CHD, however, is higher in older people. Moreover, older people are on average less physically active than younger people (Schoenborn, Adams, Barnes, Vickerie, \& Schiller, 2004). Thus, they might need special treatment to help them change their unhealthy lifestyle behaviors (Mills et al., 1996).

Behavior changes are difficult to achieve and to maintain, as studies on the long-term effects of cardiac rehabilitation demonstrate (Dusseldorp, Van Elderen, Maes, Meulman, \& Kraaij, 1999; Johnston, 1999). Even strong intentions to change one's behavior do not guarantee success (e.g., Godin \& Kok, 1996; Sheeran, 2002). Consequently, interventions focusing mainly on enhancing intentions to change behavior, as some health behavior theories suggest (e.g., Theory of Planned Behavior, Ajzen, 1991), might not be sufficient for facilitating changes of behavior. Self-regulatory skills, however, may help translating intentions into behavior. Self-regulatory skills might be addressed in interventions that aim at promoting regular physical exercise, for example self-efficacy (Lorig, \& Holman, 2003; McAuley, Courney, Rudolph, \& Lox, 1994). Another self-regulatory skill that has proven useful for behavioral change is planning (Gollwitzer, 1999; Leventhal, Singer, \& Jones, 1965). Planning is generally understood as a means of simulating behavior mentally and prospectively to be prepared for situations in which the behavior should be executed (Lachman \& Burack, 1993). It is an economic

\footnotetext{
Authors' Note: We would like to thank Jochen P. Ziegelmann for helpful comments on an earlier draft of this article. Please address correspondence to Dr. Urte Scholz, University of Zurich, Department of Psychology, Social and Health Psychology, Binzmuehlestr. 14/ Box 14, CH 8050, Zurich, Switzerland; e-mail: urte.scholz@psychologie.unizh.ch
} 
(in terms of saving time and energy) and effective way of fostering goal attainment (Gollwitzer, 1999).

Unlike self-efficacy, planning can be addressed in a 15- to 30-min intervention and therefore could be easily integrated into existing rehabilitation treatments. Age-related differences in the role of planning in health behavior change have not yet been a subject of study. This study aimed at promoting planning in CHD patients.

\section{Planning and Lifestyle Changes}

Action plans specify when, where, and how to implement an intended behavior (Gollwitzer, 1999; Leventhal et al., 1965). Action planning links behavioral responses to situational cues. Through planning, these situational cues can elicit behavioral responses automatically, that is, without conscious intent, because the situational cues specified in the plans are recognized with more ease and appropriate responses are already at hand (Gollwitzer, 1999). Action planning is understood as a process that comes into play after an intention has been formed. By comparing the behavioral effects of intentions alone to intentions plus action planning, studies have repeatedly shown that action planning leads to a higher likelihood that the behavior will be implemented than do good intentions alone (Gollwitzer \& Sheeran, 2006). The usefulness of action planning for health behavior change has been demonstrated in different domains, such as tetanus vaccination (Leventhal et al., 1965), breast self-examination (Luszczynska \& Schwarzer, 2003), physical activity (Milne, Orbell, \& Sheeran, 2002), and nutrition (Verplanken \& Faes, 1999).

Planning not only addresses the execution of an intended action. Individuals can also plan how to control unwanted distractions and how to cope with difficulties that interfere with the execution (Gollwitzer \& Schaal, 1998; Patterson \& Mischel, 1976). This coping planning (Sniehotta, Schwarzer, Scholz, \& Schüz, 2005) can be helpful in changing complex health behaviors, such as initiating and maintaining regular physical exercise (e.g., Simkin \& Gross, 1994; Sniehotta, Scholz \& Schwarzer, 2006). Coping planning is barrier focused because it includes both the anticipation of personal difficulties and barriers that may hinder a person from acting as intended and the planning of exact strategies for overcoming those barriers. Similar approaches are used in cessation programs for addictive behaviors (Marlatt, 1996). Thus, coping planning is assumed to promote the effects of action planning on behavior change. Together, these two planning skills are assumed to be helpful for successfully changing complex health behaviors. 


\section{Planning and Older Age}

Only a few studies have investigated the effects of planning on behavior change in older participants. A study by Chasteen, Park, and Schwarz (2001) demonstrated that despite the age-related decline in prospective memory, older participants benefited from action-planning interventions and were almost twice as likely as the control group to successfully perform a laboratory task. The authors concluded that the planning intervention was effective for older participants because the automatic process of behavior initiation was independent from prospective memory capacities. Thus, this study demonstrated that the automatic initiation of behavior accounts for the effects of planning on behavior for older participants as well. In the domain of health behavior change, action- and coping-planning interventions have been found to enhance physical exercise in rehabilitation patients, independent of their chronological age (Sniehotta et al., 2006; Ziegelmann, Lippke, \& Schwarzer, 2006). An action-planning intervention on older patients' engagement in activities of daily living (ADLs) after hip replacement showed that participants in the intervention group were more likely to perform ADLs than were those in the control group (Orbell \& Sheeran, 2000). Also in the area of medical adherence, the effectiveness of an action-planning intervention has been demonstrated in an older sample (Liu \& Park, 2004). The participants in the intervention group engaged almost 50\% more often in the target behavior, namely, home blood glucose monitoring, than did the two control groups. These results emphasize that planning interventions are an appropriate means to facilitate behavior change in both younger and older adults.

When implementing a theory-based intervention study, it is important to investigate the mediating mechanisms by which the intervention is assumed to unfold its effects. This includes testing whether the intervention had an effect on the theoretical determinants of behavior change and whether these effects mediate behavioral effects. If a full mediation is found, intervention effects can be accounted for by the assumed underlying psychological processes. Unspecific processes, such as social interactions, can be ruled out (Michie \& Abraham, 2004).

\section{Purpose of the Study}

The purpose of this study was threefold. First, an action-planning intervention and an action-planning-plus-coping-planning intervention were compared to each other and to a control group in terms of their effects on physical activity. Second, it tested whether changes in action planning and 
coping planning constituted mediating mechanisms of the interventions on behavior. Third, it examined whether the effects of the interventions on exercise and on planning were related to the ages of the participants.

\section{Method}

\section{Sample and Procedures}

Data collection took place in three inpatient cardiac rehabilitation centers in Germany at two measurement times. In general, patients stayed 3 weeks in the clinic and underwent a comprehensive psychoeducational and somatic treatment. The rehabilitation treatment is provided on an inpatient basis and includes daily guided physical exercise sessions supervised by a physician. In addition to the physical exercise treatment, patients attend educational courses on preventing CHD risk factors (e.g., balanced nutrition, smoking cessation), as well as stress management courses, aimed at restoration and maintenance of physical and mental health (Dietz \& Rauch, 2003). All patients of this study were instructed by their physicians to engage in regular vigorous exercise after discharge.

Each participant gave informed consent and received a personal code to match the data of the questionnaires to ensure anonymity. Participation was voluntary, and no financial compensation was offered. The first assessment (Time 1 [T1]) took place during the second week of participants' stay in the cardiac rehabilitation clinic. For the second assessment (Time 2 [T2]), 2 months after discharge, the questionnaires were mailed to the participants at home.

Participants were assigned to either a control group or one of two planning intervention groups. The planning interventions took place during the third week of the rehabilitation in the clinic. All interventions were implemented in individual sessions by trained interviewers. Interviewers assisted participants in a nondirective manner and intervened only when problems occurred to ensure that patients formulated their own personal plans that were realistic and relevant to them (cf. Sniehotta et al., 2006).

Participants of the action-planning group received a planning sheet from their interviewer and were invited to read the instructions written on the planning sheet: "Please think about the time after discharge from the rehabilitation center. When, where and how do you plan to be physically active? Please write down your plans in the following table. The more precisely, concretely, and personally you formulate your plans, the more they can help you." Participants could generate up to three action plans. 
Participants of the action-plus-coping-planning group received a planning sheet that included the same instructions as for the action plans as well as additional instructions to write down up to three coping plans. The instructions for the coping plans read, "What obstacles or barriers might interfere with the implementation of your exercise plans? How could you successfully cope with such problems? Please write down your plans in the following table. The more precisely, concretely, and personally you formulate your plans, the more they will help you."

Overall, 237 patients agreed to participate in the study. Of these, 205 (86.5\%) completed the second questionnaire 2 months after discharge. Men made up $79.5 \%$ of the respondents $(n=163)$. The mean age was 59.6 years $(S D=9.62)$, ranging from 38 to 82 years. For subsequent analyses, three age groups were established. Individuals aged 38 to $54(n=64)$ were categorized as young, from 55 to $64(n=82)$ as middle aged, and from 65 to $82(n=59)$ as old.

The majority of the participants were married $(82.4 \% ; n=169), 6.3 \%$ $(n=13)$ were divorced, $6.3 \%(n=13)$ were single, $3.4 \%(n=7)$ were widowed, and $3(1.5 \%)$ did not indicate their marital status. Of all participants, 19.5\% $(n=40)$ attended school for 13 years; $23.9 \%(n=49)$ reported 12 years of schooling; $18.5 \%(n=38), 10$ years; $32.7 \%(n=67)$, 9 years; and 11 participants $(5.5 \%)$ did not report their years of schooling. Only $44.4 \%(n=91)$ of the patients were employed. The control group consisted of 83 participants, the action-planning group of 71 , and the action-plus-copingplanning group of 51 .

Cell sizes of the three groups turned out to be unequal because of the assignment procedure. Consecutively, participants were first assigned to the control group, then to the action planning, and then to the combined actionplanning-plus-coping-planning group. Each of the trained interviewers started with the control group when making initial contacts with patients. The group sizes were unequal because the interviewers saw an unequal number of patients.

\section{Measures}

The response format for all scales was a 4-point Likert-type scale that ranged from 1 (not at all true) to 4 (exactly true).

Behavioral intentions were assessed at $\mathrm{T} 1$ for the time after discharge from the rehabilitation center and were measured by six items specific to physical exercise (Sniehotta, Scholz, \& Schwarzer, 2005). The stem "I intend to . . ." was followed by recommended activities, for example, ". . . be 
physically active regularly several times per week." $\mathrm{T} 1$ behavioral intentions had a Cronbach's alpha $=.80, M=3.41, S D=.51$.

Action planning was assessed at $\mathrm{T} 1$ and $\mathrm{T} 2$ with a four-item scale (Sniehotta, Scholz, et al., 2005). The items consisted of the stem "I have made a detailed plan regarding . . .," which was followed by (a) ". . . when to exercise," (b) ". . . . where to exercise," (c) ". . . how to exercise," and (d) ". . . how often to exercise." Action planning at T1 had a Cronbach's alpha of .93, $M=3.20, S D=.94$. At T2, Cronbach's alpha was .95, $M=3.23, S D=.89$.

Coping planning, which was assessed at $\mathrm{T} 1$ and $\mathrm{T} 2$ with a five-item scale (Sniehotta, Schwarzer et al., 2005), included the same stem as the action planning items: "I have made a detailed plan regarding . . .," which was followed by five items, such as ". . . what to do if something interferes with my plans." Cronbach's alpha of coping planning at T1 was . $90, M=2.5$, $S D=.90$ and at $\mathrm{T} 2$ it was $.90, M=2.70, S D=.86$.

For the assessment of physical exercise, a subset of the International Physical Activity Questionnaire (Booth, 2000) was used. At T1, physical exercise was assessed retrospectively for the time before the acute cardiac event. Participants were asked how often per week they had engaged in vigorous exercise such as swimming, biking, running, or power walking. Because the question also comprised power walking, it can be regarded as appropriate for older people. The answers were summed up to an overall score indicating the average workout frequency per week. At T2, participants were asked how often they had engaged on average in these activities during the past 4 weeks. The average frequency of (untransformed) physical exercise at $\mathrm{T} 1$ was $M=.90, S D=1.78$, and at $\mathrm{T} 2$ it was $M=2.47$, $S D=2.5$. The distributions of physical exercise scores at both times were skewed because a high proportion of the respondents were inactive. To smooth the distributions and approximate a normal curve, a logarithmic transformation was applied (Tabachnick \& Fidell, 2001).

\section{Results}

\section{Attrition Analysis and Randomization Check}

Comparing participants who did not complete $\mathrm{T} 2$ with those who did yielded no significant differences in age, $\mathrm{T} 1$ intentions, $\mathrm{T} 1$ action planning, $\mathrm{T} 1$ coping planning, and $\mathrm{T} 1$ physical exercise. Likewise, no differences in gender emerged. For the control group and the two intervention groups, no baseline differences were found for age, sex, sociodemographics, behavioral intentions, action planning, coping planning, or physical activity. 


\section{Descriptives}

Age was correlated with action planning at neither $\mathrm{T} 1$ nor $\mathrm{T} 2$. There was a significant positive correlation between age and coping planning at $\mathrm{T} 1$, but not at $\mathrm{T} 2$, which indicates that older participants engaged in more coping planning than did younger participants before the intervention. Furthermore, physical activity at $\mathrm{T} 1$ was not significantly associated with age, whereas the hypothesized negative relationship was found at T2. Table 1 displays correlations between all variables of the study. Because intentions at $\mathrm{T} 1$ were significantly associated with physical exercise at $\mathrm{T} 1$ and at $\mathrm{T} 2$, it was included as a covariate in subsequent analyses.

Table 1

Intercorrelations of Age, Planning, Intentions, and Physical Exercise

\begin{tabular}{llllllll}
\hline 1 & 2 & 3 & 4 & 5 & 6 & 7 \\
\hline
\end{tabular}

1. Age

2. Time 1 action planning

3. Time 2 action planning

4. Time 1 coping planning

5. Time 2 coping planning

6. Time 1 intentions

7. Time 1 physical exercise

8. Time 2 physical exercise

$\begin{array}{clllllll}.09 & & & & & & \\ -.05 & .30^{* *} & & & & & \\ .23 * * & .51 * * & .18^{* *} & & & & \\ .09 & .25 * * & .61 * * & .30 * * & & & \\ .05 & .48 * * & .31 * * & .37 * * & .33 * * & & \\ .05 & .21 * * & .06 & .37 * * & .01 & .24 * * & \\ -.15 * & .16^{*} & .30 * * & .13 & .13 & .23 * * & .33 * *\end{array}$

$* p<.05 .{ }^{* *} p<.01$.

\section{Predicting Change in Physical Exercise}

To test the effects of the intervention and of age on physical exercise, a repeated measures ANCOVA was conducted, with treatment groups and age groups as factors and intentions at $\mathrm{T} 1$ as covariate. The Time $\times$ Treatment interaction and the Time $\times$ Age Groups interaction were significant, $F(2,195)=3.34, p<.05, \eta^{2}=.03$ and $F(2,195)=4.30, p<.05, \eta^{2}=.04$, respectively. The interaction between time and intervention shows that the control group had the least increase in physical exercise over time, whereas the action-planning-plus-coping-planning group attained the highest increase. Pairwise comparisons revealed that only the difference between the control group and the action-planning-plus-coping-planning group reached statistical significance, $p=.01$. 


\section{Figure 1}

\section{Time $\times$ Treatment Interaction (top). Time $\times$} Age Group Interactions (bottom).

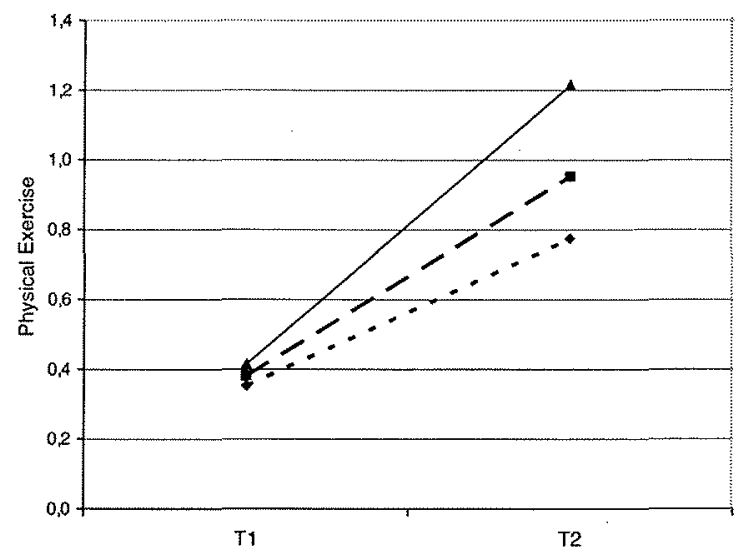

$\rightarrow-$ AP\&CP Group $\rightarrow-$ AP Group - - CO Group

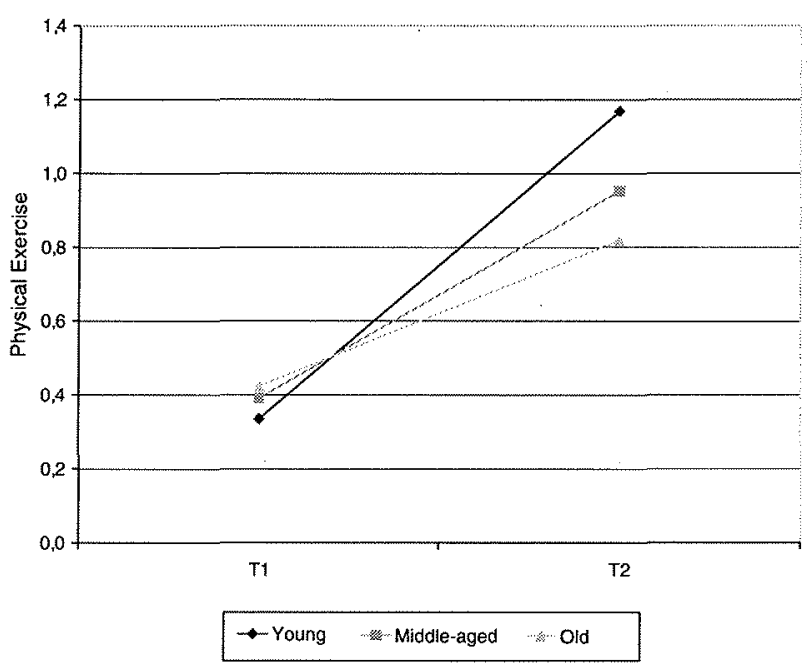

Note: $\mathrm{CO}=$ Control Group, AP = Action-Planning Group, AP\&CP = Action-Planning and Coping- Planning Group. Y-Axes Represent Average Frequency of Physical Exercise Per Week.

The interaction between time and age group demonstrates that the youngest participants had the highest increase in physical exercise over time, whereas the oldest had the lowest. A regression analysis with age as continuous variable also resulted in a highly significant effect of age on the change score of physical exercise, $\beta=-.19, p<.01$. Figure 1 , top and bottom, displays the interaction effects. 


\section{Predicting Change in Action Planning and Coping Planning}

To test whether action planning and coping planning were enhanced by the treatment, two repeated measures ANCOVAs were conducted, with action planning and coping planning as dependent variables. Treatment and age groups were included as factors, and intentions at T1 were included as covariate. The results for action planning showed that neither the Time $\times$ Treatment nor the Time $\times$ Age Groups interaction effects reached statistical significance, $F(2,194)=2.33, p>.10$, and $F(2,194)=2.21, p>.10$, respectively. Another picture emerged when coping planning was considered as a dependent variable. Here, the Time $\times$ Treatment interaction was significant, $F(2,195)=.42, p<.05, \eta^{2}=.03$. This interaction indicates that the control group had the lowest change in coping planning over time, and the action-plus-coping planning group had the highest change. Pairwise comparisons revealed that both the control group and the action-planning group differed significantly from the action-planning-plus-coping-planning group, $p<.05$. The control group and the action-planning group, however, did not differ significantly in terms of change in coping planning.

The Time $\times$ Age Groups interaction was also significant, $F(2,195)=5.03$, $p<.01, \eta^{2}=.05$. The youngest participants showed the lowest initial levels of coping planning and increased over time, T1: $M=2.19, S D=.11$; T2: $M=2.57, S D=.10$. The middle-aged participants started from a higher level than the younger participants and also showed an increase, $\mathrm{T} 1: M=2.48$, $S D=.09 ; \mathrm{T} 2: M=2.89, S D=.09$. The oldest participants had the highest level of coping planning at $\mathrm{T} 1$, and their level remained relatively stable over time, T1: $M=2.80, S D=.11 ; \mathrm{T} 2: M=2.70, S D=.11$.

Post hoc analyses revealed that the differences in change in coping planning across age groups were significant between the young and old, $p<.01$, and between the middle-aged and the old, $p<.01$, but not between young and middle-aged participants.

\section{Mediator Analyses}

To test whether action planning and coping planning represent mediating factors between the intervention on exercise change, three conditions need to be satisfied (Baron \& Kenny, 1986). First, the intervention must have a significant effect on exercise change. Second, the intervention must have a significant effect on change in action planning and coping planning. Third, the inclusion of the change score of action planning and coping planning as 
a covariate in the analyses of the intervention on exercise change must lead to a substantial decrease of the intervention effect. The covariates need to be significantly associated with exercise change. For action planning, the second condition was not fulfilled. Therefore, the third step of the mediator analyses was conducted for coping planning only.

The difference score of coping planning was included in the repeated measures ANCOVA as a covariate with treatment and age groups as factors, and intentions at T1 as the second covariate. A significant Time $\times$ Change in Coping Planning interaction emerged, $F(1,194)=8.73, p<.01, \eta^{2}=.04$. The formerly significant interaction of Time $\times$ Treatment decreased to a nonsignificant level, $F(2,194)=2.38, p=.10$, and was thus fully mediated. Furthermore, the Time $\times$ Age Groups interaction decreased to $F(2,194)=$ $3.21, p=.04$ and was thus partially mediated.

\section{Discussion}

The results of this study demonstrate the effectiveness of a minimal-effort intervention superimposed on a comprehensive traditional rehabilitation program. Action and coping planning enhanced physical activity in all three age groups. The investigation of the mediating mechanisms, however, resulted in age differences on action planning and coping planning. Although no age differences were observed in action planning, older participants reported higher baseline levels of coping planning than younger people did. There are several possible explanations for this finding. For example, coping planning is more experience based than is action planning because the former refers explicitly to a person's difficulties and barriers and how to overcome them (Scholz, Sniehotta, \& Schwarzer, 2005; Sniehotta, Schwarzer, et al., 2005). With increasing age, more and more challenges are beyond one's control (Scholnick \& Friedman, 1993). Therefore, older people gain more experience with difficulties and barriers that might hinder goal attainment. The adaptive potential to deal with such difficulties in older age, however, should not be underestimated (Brandtstädter \& Renner, 1990). Because coping planning is a means to reach one's goal despite the presence of difficulties, older people might have developed more coping planning skills than had younger ones. Furthermore, because of the increased amount of resource loss that older people experience compared with younger ones (Baltes, 1997), older individuals might focus on the conservation of their resources and therefore engage more in avoiding losses than younger ones do. Anticipating difficulties and planning how to master them help people prepare better for challenging situations that may otherwise jeopardize their resources. Thus coping planning 
may serve as a compensatory strategy, being used more frequently by older people (Ziegelmann, Lippke, \& Schwarzer, 2006).

The planning interventions moved younger and middle-aged participants up to the same level of coping planning that the older participants had attained before the interventions. The increase in coping planning mediated the effect of the intervention on physical exercise. Thus, enhancing younger and middle-aged peoples' coping planning skills led to an improvement of their physical exercise levels. For older participants, however, the baseline level was already quite high, so they did not report an increase in coping planning. Thus older participants' potential to further optimize their coping planning skills might be lower than that of younger ones because older people's higher baseline level meant they had already optimized their self-regulatory skills. Likewise, older individuals' potential to increase their physical exercise to the level of younger or middle-aged people might be limited because of physical constraints (Huang et al., 1998). However, because there was a significant rise in physical exercise for older participants as well, and because this age effect on physical exercise change was at least partly mediated by coping planning change, it might also be that, not the amount of coping planning, but the quality of the plans was influenced by the coping-planning intervention in older participants. Moreover, one might speculate that, for example, older people benefit more from the social interaction with the interviewer than younger ones (Ziegelmann et al., 2006).

Planning is a postintentional process succeeding a motivational decision process (Gollwitzer, 1999). Investigating postintentional processes such as coping planning, which can be used as a compensatory strategy, might be biased in a study with a unique sample such as CHD patients. Younger individuals with a chronic disease might be more likely to use compensatory strategies than would healthy young people. Therefore, in healthy individuals, age differences in coping planning might be even larger because older people are generally more likely to lack resources than younger ones. This might not have been the case in this specific sample of men and women with CHD, because CHD represents a condition that limits individual resources.

No age differences for action planning have been found. Likewise, no effects of the action-planning intervention on change in action planning were found. On average, action planning at baseline was already quite high, $M=3.2$, $S D=.94$, on a scale ranging from 1 to 4 . It may be that encouraging patients to engage in action planning is already included in the psychoeducational syllabus of the rehabilitation centers that the patients participate in.

This study has some limitations. First, data on physical activity were self-reported. Although most of the literature on the relationship between 
physical exercise and risk of CHD rely on self-report measures, and although the International Physical Activity Questionnaire (Booth, 2000) is a thoroughly validated measure, potential biases can be assumed. Future studies might want to include objective data. Second, the time between baseline measurement and follow-up was rather short in terms of the investigation of long-term effects. It should be noted, however, that in studies of the effects of planning on behavior change, an even shorter time frame of 2 to 4 weeks is rather common (cf. Gollwitzer \& Sheeran, 2006). Moreover, the examination of the mediating mechanisms might have benefited from a shorter follow-up, so that a significant effect of action planning may have emerged also only 2 weeks after the intervention. Third, the effect sizes of the intervention effects were rather small.

Even with these limitations, the findings are promising and suggest that coping planning and action planning should be explored more fully in the context of a minimal intervention strategy for promoting physical activity among individuals with chronic illnesses.

The clinical relevance of these results, however, is high. All participants underwent a 3- to 4-week inpatient treatment, participating in a comprehensive psychoeducational program that included daily supervised exercise classes, nutrition management, relaxation techniques, and so on (Dietz \& Rauch, 2003). The present intervention study represents a parsimonious treatment on top of this comprehensive 3-week treatment. It lasted on average less than $20 \mathrm{~min}$. The result that a brief and cost-effective planning intervention was successful in changing physical activity is to be highlighted. Still, further research is needed that focuses on the long-term effects of these planning interventions. Moreover, research should further address possible age-related advantages of the better coping planning skills in older adults.

\section{References}

Ades, P. A. (2001). Cardiac rehabilitation and secondary prevention of coronary heart disease. New England Journal of Medicine, 345, 892-902.

Ajzen, I. (1991). The theory of planned behavior. Organizational Behavior and Human Decision Processes, 50, 179-211.

Anderson, R. N., \& Smith, B. L. (2005). Deaths: Leading causes for 2002. National Vital Statistics Reports, 53(17), 1-89.

Baltes, P. B. (1997). On the incomplete architecture of human ontogeny: Selection, optimization, and compensation as foundation of developmental theory. American Psychologist, $52,366-380$.

Baron, R. M., \& Kenny, D. A. (1986). The mediator-moderator variable distinction in social psychological research: Conceptual, strategic, and statistical considerations. Journal of Personality and Social Psychology, 51, 1173-1182. 
Booth, M. L. (2000). Assessment of physical activity: An international perspective. Research Quarterly for Exercise and Sport, 71, 114-120.

Brandtstädter, J., \& Renner, G. (1990). Tenacious goal pursuit and flexible goal adjustment: Explication and age-related analysis of assimilative and accommodative strategies of coping. Psychology and Aging, 5, 58-67.

Chasteen, A. L., Park, D. C., \& Schwarz, N. (2001). Implementation intentions and facilitation of prospective memory. Psychological Science, 12, 457-461.

Dietz, R., \& Rauch, B. (2003). Leitlinie zur Diagnose und Behandlung der chronischen koronaren Herzerkrankung der Deutschen Gesellschaft für Kardiologie- Herz- und Kreislaufforschung (DGK) [Guidelines for the diagnosis and treatment of chronic coronary heart disease. Issued by the executive committee of the German Society of Cardiology- Heart- and Circulation Research in cooperation with the German Society for Prevention and Rehabilitation of Cardiac Diseases and the German Society for Thoracic and Cardiovascular Surgery]. Zeitschrift für Kardiologie, 92, 501-521.

Dusseldorp, E., Van Elderen, T., Maes, S., Meulman, J., \& Kraaij, V. (1999). A meta-analysis of psychoeducational programs for coronary heart disease patients. Health Psychology, $18,506-519$.

Godin, G., \& Kok, G. (1996). The theory of planned behavior: A review of its applications to health-related behaviors. American Journal of Health Promotion, 11, 87-97.

Gollwitzer, P. (1999). Implementation intentions: Strong effects of simple plans. American Psychologist, 54, 493-503.

Gollwitzer, P. M., \& Schaal, B. (1998). Metacognition in action: The importance of implementation intentions. Personality and Social Psychology Review, 2, 124-136.

Gollwitzer, P. M., \& Sheeran, P. (2006). Implementation intentions and goal achievement: A meta-analysis of effects and processes. Advances in Experimental Social Psychology, 38, 69-119.

Huang, Y., Macera, C. A., Blair, S. N., Brill, P. A., Kohl, H. W., \& Kronenfeld, J. J. (1998). Physical fitness, physical activity, and functional limitation in adults aged 40 and older. Medicine and Science in Sports and Exercise, 30, 1430-1435.

Johnston, D. W. (1999). Lifestyle changes after a myocardial infarction. Heart, 82, 243-244.

Jolliffe, J. A., Rees, K., Taylor, R. S., Thompson, D., Oldridge, N., \& Ebrahim, S. (2003). Exercise-based rehabilitation for coronary heart disease. Available from http://www.mrw .interscience.wiley.com/cochrane/clsysrev/articles/CD001800/frame.html

Lachman, M. E., \& Burack, O. R. (1993). Planning and control processes across the life span: An overview. International Journal of Behavioral Development, 16, 131-143.

Leventhal, H., Singer, R., \& Jones, S. (1965). Effects of fear and specificity of recommendation upon attitudes and behavior. Journal of Personality and Social Psychology, 2, 20-29.

Liu, L. L., \& Park, D. C. (2004). Aging and medical adherence: The use of automatic processes to achieve effortful things. Psychology and Aging, 19, 318-325.

Lorig, K. R., \& Holman, H. R. (2003). Self-management education: History, definition, outcomes, and mechanisms. Annals of Behavioral Medicine, 26, 1-7.

Luszczynska, A., \& Schwarzer, R. (2003). Planning and self-efficacy in the adoption and maintenance of breast self-examination: A longitudinal study on self-regulatory cognitions. Psychology and Health, 18, 93-108.

McAuley, E., Courneya, K. S., Rudolph, D. L., \& Lox, C. L.. (1994). Enhancing exercise adherence in middle-aged males and females. Preventive Medicine, 23, 498-506.

Marlatt, G. A. (1996). Taxonomy of high-risk situations for alcohol relapse: Evolution and development of a cognitive-behavioral model. Addiction, 91, 37-50. 
Merrill, S. S., \& Verbrugge, L. M. (1999). Health and disease in midlife. In S. Willis \& J. D. Reid (Eds.), Life in the middle: Psychological and social development (pp. 77-103). San Diego, CA: Academic Press.

Michie, S., \& Abraham, C. (2004). Interventions to change health behaviors: Evidence-based or evidence-inspired? Psychology \& Health, 19, 29-51.

Mills, K. M., Stewart, A. L., King, A. C., Roitz, K., Sepsis, P. G., Ritter, P. L., et al. (1996). Factors associated with enrollment of older adults into a physical activity promotion program. Journal of Aging \& Health, 8, 96-113.

Milne, S., Orbell, S., \& Sheeran, P. (2002). Combining motivational and volitional interventions to promote exercise participation: Protection motivation theory and implementation intentions. British Journal of Health Psychology, 7, 163-184.

Orbell, S., \& Sheeran, P. (2000). Motivational and volitional processes in action initiation: A field study of the role of implementation intentions. Journal of Applied Social Psychology, 30, 780-797.

Patterson, C. J., \& Mischel, W. (1976). Effects of temptation-inhibiting and task-facilitating plans on self-control. Journal of Personality and Social Psychology, 33, 209-217.

Schoenborn, C. A., Adams, P. F., Barnes, P. M., Vickerie, J. L., \& Schiller, J. S. (2004). Health behaviors of adults: United States, 1999-2001. National Center for Health Statistics. Vital Health Stat 10(219). Retrieved June 19, 2007, from http://www.cdc.gov/nchs/data/series/ sr_10/sr10_219.pdf

Scholnick, E. K., \& Friedman, S. L. (1993). Planning in context: Developmental and situational considerations. International Journal of Behavioral Development, 16, 145-167.

Scholz, U., Sniehotta, F. F., \& Schwarzer, R. (2005). Predicting physical exercise in cardiac rehabilitation: The role of phase-specific self-efficacy beliefs. Journal of Sport \& Exercise Psychology, 27, 135-151.

Sheeran, P. (2002). Intention-behavior relations: A conceptual and empirical review. In M. Hewstone \& W. Stroebe (Eds.), European review of social psychology (Vol. 12, pp. 1-36). Chichester, England: Wiley.

Simkin, L. R., \& Gross, A. M. (1994). Assessment of coping with high-risk situations for exercise relapse among healthy women. Health Psychology, 13, 274-277.

Smith, S. C., Jr., Blair, S. N., Bonow, R. O., Brass, L. M., Cerqueira, M. D., Dracup, K., et al. (2001). AHA/ACC Guidelines for preventing heart attack and death in patients with atherosclerotic cardiovascular disease: 2001 update. A statement for healthcare professionals from the American Heart Association and the American College of Cardiology. Circulation, 104, 1577-1579.

Sniehotta, F. F., Scholz, U., \& Schwarzer, R. (2005). Bridging the intention-behavior gap: Planning, self-efficacy, and action control in the adoption and maintenance of physical exercise. Psychology \& Health, 20, 143-160.

Sniehotta, F. F., Scholz, U., \& Schwarzer, R. (2006). Action plans and coping plans for physical exercise: A longitudinal intervention study in cardiac-rehabilitation. British Journal of Health Psychology, 11, 23-37.

Sniehotta, F. F., Schwarzer, R., Scholz, U., \& Schüz, B. (2005). Action planning and coping planning for long-term lifestyle change: Theory and assessment. European Journal of Social Psychology, 35, 565-576.

Tabachnick, B. G., \& Fidell, L. S. (2001). Using multivariate statistics (4th ed.). Boston, MA: Allyn \& Bacon.

Thompson, P. D., Buchner, D., Piña, M. D., Balady, G. J., Williams, M. A., Marcus, B. H., et al. (2003). Exercise and physical activity in the prevention and treatment of atherosclerotic cardiovascular disease. Circulation, 107, 3109-3116. 
Verplanken, B., \& Faes, S. (1999). Good intentions, bad habits and the effects of forming implementation intentions on behavior and cognition. European Journal of Social Psychology, 29, 591-604.

Weidner, G. (2001). Gender and cardiovascular health. In N. J. Smelser \& P. B. Baltes (Eds.), International encyclopedia of the social and behavioral sciences (Vol. 20, pp. 5904-5907). Oxford, UK: Elsevier Science.

Ziegelmann, J. P., Lippke, S., \& Schwarzer, R. (2006). Adoption and maintenance of physical activity: Planning interventions in young, middle-aged, and older adults. Psychology \& Health, 21, 145-163. 